\title{
Identification of Secondary Structure of Extracellular Signal Regulated Kinase (ERK) Interacting Proteins and Their Domain: An in Silico Study
}

\author{
Kurrey Khuleshwari, Paramanik Vijay* \\ Cellular and Molecular Neurobiology \& Drug Targeting Laboratory, Department of Zoology, Indira Gandhi National Tribal \\ University, Amarkantak (MP), (MP)-484 887, India \\ Email: *vijayparamanik@gmail.com
}

How to cite this paper: Khuleshwari, K. and Vijay, P. (2021) Identification of Secondary Structure of Extracellular Signal Regulated Kinase (ERK) Interacting Proteins and Their Domain: An in Silico Study. World Journal of Neuroscience, 11, 67-89. https://doi.org/10.4236/wjns.2021.111007

Received: November 29, 2020

Accepted: February 23, 2021

Published: February 26, 2021

Copyright $\odot 2021$ by author(s) and Scientific Research Publishing Inc. This work is licensed under the Creative Commons Attribution International License (CC BY 4.0).

http://creativecommons.org/licenses/by/4.0/

\begin{abstract}
ERK is involved in multiple cell signaling pathways through its interacting proteins. By in silico analysis, earlier we have identified 22 putative ERK interacting proteins namely; ephrin type-B receptor 2 isoform 2 precursor (EPHB2), mitogen-activated protein kinase 1 (MAPK1), interleukin-17 receptor D precursor (IL17RD), WD repeat domain containing 83 (WDR83), tescalcin (Tesc), mitogen-activated protein kinase kinase kinase 4 (MAPP3K4), kinase suppressor of Ras2 (KSR2), mitogen-activated protein kinase kinase 6 (MAP3K6), UL16 binding protein 2 (ULBP2), UL16 binding protein 1 (ULBP1), dual specificity phosphatase 14 (DUSP14), dual specificity phosphatase 6 (DUSP6), hyaluronan-mediated motility receptor (RHAMM), kinase $\mathrm{D}$ interacting substrate of $220 \mathrm{kDa}$ (KININS220), membrane-associated guanylate kinase (MAGI3), phosphoprotein enriched in astrocytes 15 (PEA15), typtophenyl-tRNA synthetase, cytoplasmic (WARS), dual specificity phosphatase 9 (DUSP9), mitogen-activated protein kinase kinase kinase 1 (MAP3K1), UL16 binding protein 3 (ULBP3), SLAM family member 7 isoform a precursor (SLAMMF7) and mitogen activated protein kinase kinase kinase 11 (MAP3K11) (Table 1). However, prediction of secondary structure and domain/motif present in aforementioned ERK interacting proteins is not studied. In this paper, in silico prediction of secondary structure of ERK interacting proteins was done by SOPMA and motif/domain identification using motif search. Briefly, SOPMA predicted higher random coil and alpha helix percentage in these proteins (Table 2) and motif scan predicted serine/threonine kinases active site signature and protein kinase ATP binding region in majority of ERK interacting proteins. Moreover, few have com-
\end{abstract}


monly dual specificity protein phosphatase family and tyrosine specific protein phosphatase domains (Table 3). Such study may be helpful to design engineered molecules for regulating ERK dependent pathways in disease condition.

\section{Keywords}

ERK, Secondary Structure, Motif Scan, Random Coils, Alpha Helix, Protein Kinases

\section{Background}

Prediction of protein function is one of the key tasks of bioinformatics. The prediction of protein function is dependent on secondary structure and their amino acid sequence. Further, use of bioinformatics has revealed construction blocks of several proteins in recent years. The construction of 3D protein structure and prediction of biological function is increasing day by day. The region of protein actually interacted and involved in biological function is called domain/motifs. Therefore, prediction of protein structure is highly useful. However, it gives additional trouble to save protein structures and their features based on domain/motifs.

Usually most of the signaling transduction pathways use protein kinases to phosphorylate target protein by phosphorylation process. However, how protein kinases are able to achieve specificity needed to control several cellular functions remains a significant question [1]. Nonetheless, many distinct stimuli can activate the protein kinase cascade which phosphorylates versatile molecule ERK and controls multiple cellular signals [2]. Moreover, it is still uncertain how single ERK molecule is evolved to control comprehensive arrays of cellular and molecular mechanism. One of the possibilities is the recruitment of ERK interacting proteins. After interaction with these proteins, ERK controls array of cellular activity like cell proliferation, cell migration, survival, neuronal growth and neurotransmission. In addition, ERK dependent pathway is influenced by various stimulations such as growth, cytokines, viruses, second messengers, carcinogens and transforming agents [3] [4].

The protein kinases family is huge with lots of variation. However, a very few differ in size, sub cellular distribution and mode of action. The catalytic accuracy of protein kinases is very critical as they work as regulatory molecules for biological functions. Further, domain/motif of a protein serves as actual site of interaction for the manifestation of a specific biological function. Hence prediction of domain/motif or sites of proteins has been done using bioinformatics tools over the past decade. The prediction of domain/motif actually helps researchers to recognize proteins binding sites, secondary structures, 3D structure and building blocks [5]. However, there are only a few techniques which are used for general investigation of significant motifs in protein structures [6]. 
A protein attains various conformations to perform diverse functions. Further, different conformations of proteins actually determine possibility of specific motifs leading to several functions. Further, in silico identification of ERK interacting proteins and physicochemical analysis was reported earlier [3] (Table 1). These were namely; ephrin type-B receptor 2 isoform 2 precursor (EPHB2), mitogen-activated protein kinase 1 (MAPK1), interleukin-17 receptor D precursor (IL17RD), WD repeat domain containing 83 (WDR83), tescalcin (Tesc), mitogen-activated protein kinase kinase kinase 4 (MAPP3K4), kinase suppressor of Ras2 (KSR2), mitogen-activated protein kinase kinase 6 (MAP3K6), UL16 binding protein 2 (ULBP2), UL16 binding protein 1 (ULBP1), dual specificity phosphatase 14 (DUSP14), dual specificity phosphatase 6 (DUSP6), hyaluronan-mediated motility receptor (RHAMM), kinase D interacting substrate of $220 \mathrm{kDa}$ (KININS220), membrane-associated guanylate kinase (MAGI3), phosphoprotein enriched in astrocytes 15(PEA15), typtophenyl-tRNA synthetase,

Table 1. ERK interacting proteins.

\begin{tabular}{|c|c|c|c|}
\hline S. N. & $\begin{array}{l}\text { NCBI Reference } \\
\text { Sequence }\end{array}$ & $\begin{array}{l}\text { Gene } \\
\text { Name }\end{array}$ & Protein Name \\
\hline 1 & NP_004433 & EPHB2 & ephrin type-B receptor 2 isoform 2 precursor \\
\hline 2 & NP_620407.1 & MAPK1 & mitogen-activated protein kinase 1 \\
\hline 3 & NP_060033.3 & IL17RD & interleukin-17 receptor $\mathrm{D}$ precursor \\
\hline 4 & NP_001093207.1 & WDR83 & WD repeat domain-containing protein 83 \\
\hline 5 & AAH15221.1 & TESC & tescalcin \\
\hline 6 & AAI36277.1 & MAP3K4 & mitogen-activated protein kinase kinase kinase 4 \\
\hline 7 & Q6VAB6.2 & KSR2 & Kinase suppressor of Ras 2 \\
\hline 8 & EAX07758.1 & MAP3K6 & mitogen-activated protein kinase kinase kinase 6 \\
\hline 9 & AAQ89028.1 & ULBP2 & UL16 binding protein 2 \\
\hline 10 & AAK13081.1 & ULBP1 & UL16 binding protein 1 \\
\hline 11 & CAG38739.1 & DUSP14 & dual specificity phosphatase 14 \\
\hline 12 & BAA34369.1 & DUSP6 & dual specificity phosphatase 6 \\
\hline 13 & AAI08905.1 & RHAMM & hyaluronan-mediated motility receptor \\
\hline 14 & NP_065789.1 & KIDINS220 & kinase D-interacting substrate of $220 \mathrm{kDa}$ \\
\hline 15 & NP_001136254.1 & MAGI3 & membrane-associated guanylate kinase \\
\hline 16 & CAG46533.1 & PEA15 & phosphoprotein enriched in astrocytes 15 \\
\hline 17 & AAH95453.1 & WARS & tryptophanyl-tRNA synthetase, cytoplasmic \\
\hline 18 & AAH60837.1 & DUSP9 & dual specificity phosphatase 9 \\
\hline 19 & NP_005912.1 & MAP3K1 & mitogen-activated protein kinase kinase kinase 1 \\
\hline 20 & AAK13083.1 & ULBP3 & UL16 binding protein 3 \\
\hline 21 & NP_067004.3 & SLAMF7 & SLAM family member 7 isoform a precursor \\
\hline 22 & NP_002410.1 & MAP3K11 & mitogen-activated protein kinase kinase kinase 11 \\
\hline
\end{tabular}


cytoplasmic (WARS), dual specificity phosphatase 9 (DUSP9), mitogen-activated protein kinase kinase kinase 1 (MAP3K1), UL16 binding protein 3 (ULBP3), SLAM family member 7 isoform a precursor (SLAMMF7) and mitogen activated protein kinase kinase kinase 11 (MAP3K11). Majority of these are kinase and interact with ERK. Hence, they may contain similar secondary structures and domain/motifs. Moreover, no study of secondary structure and domain/motif is available about ERK interacting proteins. Hence, it promotes us to identify secondary structure and prediction of domain/motif of ERK interacting proteins using in silico. Such studies are useful to understand ERK dependent pathways and helpful to design engineered molecules for regulating them in disease situation.

\section{Materials and Methods}

For identification of secondary structure of ERK interacting proteins were retrieved in earlier study (Table 1) [3]. Thereafter, secondary structure of these protein were done by Self-Optimized Prediction Method with Alignment (SOPMA) (https://npsa-prabi.ibcp.fr/NPSA/npsa_sopma.html) using aa sequence of protein in FASTA format (Figure 1).

For identification of domain/motif search among ERK interacting protein, motif search, (https://www.genome.jp/tools/motif) a computer-based database was used. There is a choice of computer-based application available for identification of motifs. These were most frequently characterized by a single function namely prediction of phosphorylation sites [7]. In this paper, PROSITE was used for documentation of motif and domain of proteins. PROSITE is an annotated collection of protein families and domains classifying motif descriptors. PROSITE's motif descriptors are either prosite patterns (ProPat) or prosite profiles (ProPro) received from various alignments of homologous sequences. ProPat are descriptors of qualitative motifs. As the sequence

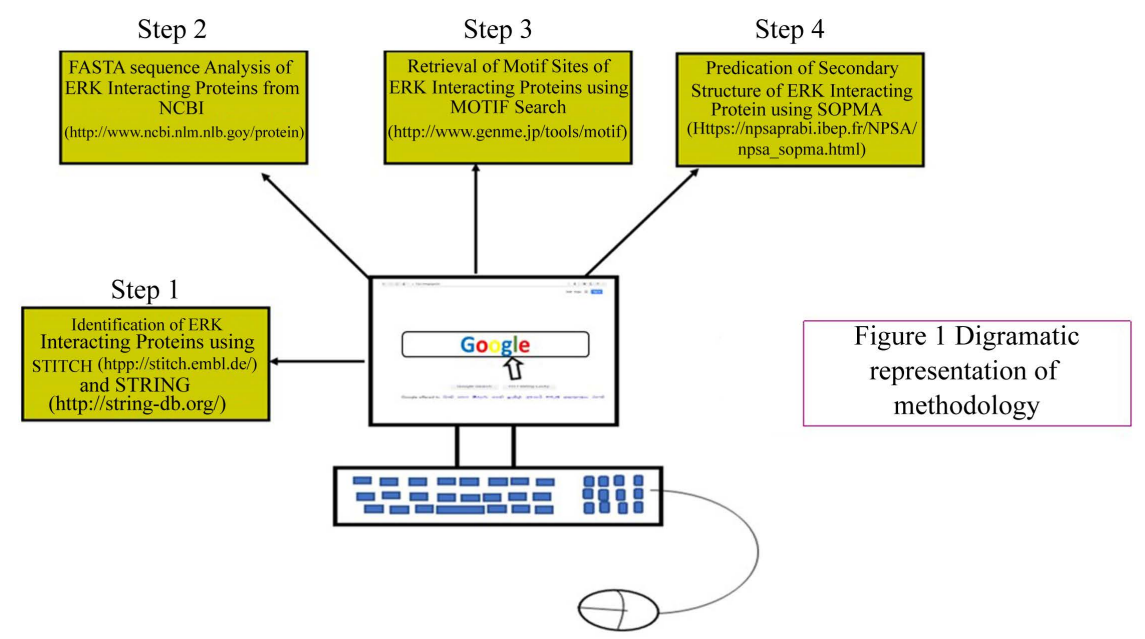

Figure 1. Schematic representation of procedure used for identification of secondary structure of ERK interacting proteins and prediction of their domain/motif. 
of biologically significant motifs is evolutionarily preserved, a multiple alignment of them can be decreased to an expression of agreement called regular expression or pattern. Every location of such a pattern can be taken up by any residue from a given set of acceptable substances, and a variable number of times within a specified spectrum for variable number of times within a specified spectrum. In addition, ProPro is a quantitative protein domain demonstration. ProPat is more complicated. Usually the ProPro corresponds with the protein domain [8].

\section{Results and Discussion}

In this study, out of 22 ERK interacting proteins; 12 proteins namely; EPHB2, MAPK1, WDR83, TESC, MAP3K4, DUSP14, KSR2, MAP3K6, MAGI3, WARS, MAP3K1 and MAP3K11 showed both ProPat and ProPro. The highest number of ProPat was found in EPHB2. Further, 6 proteins namely; IL17RD, DUSP6, KIDINS220, PEA15, DUSP9 and SLAMF7 interacting proteins showed only ProPro. However, remaining 4 ERK interacting proteins namely; ULBP2, ULBP1, RHAMM and ULBP3 do not show both ProPat and ProPro. Among above mentioned 12 proteins, 6 interacting proteins viz, MAPK1, MAP3K4, KSR2, MAP3K6, MAP3K1 and MAP3K11 showed serine/threonine kinases active site signature and protein kinase ATP binding region signature domain commonly in their pattern whereas remaining namely EPHB2, WDR83, TESC, DUSP14, MAGI3 and WARS showed different protein kinase protein family domain pattern. In addition, in case of ProPro 7 proteins namely; EPHB2, MAPK1, MAP3K4, KSR2, MAP3K6, MAP3K1 and MAP3K11, indicated protein kinase domain profile commonly and other 3 proteins namely; DUSP9, DUSP14 and DUSP6 represented commonly dual specificity protein phosphatase family profile and tyrosine specific protein phosphatase family profile. The details of ProPat and Pro Pro are listed in Table 3 and Figures 1-10 (Supplementary materials).

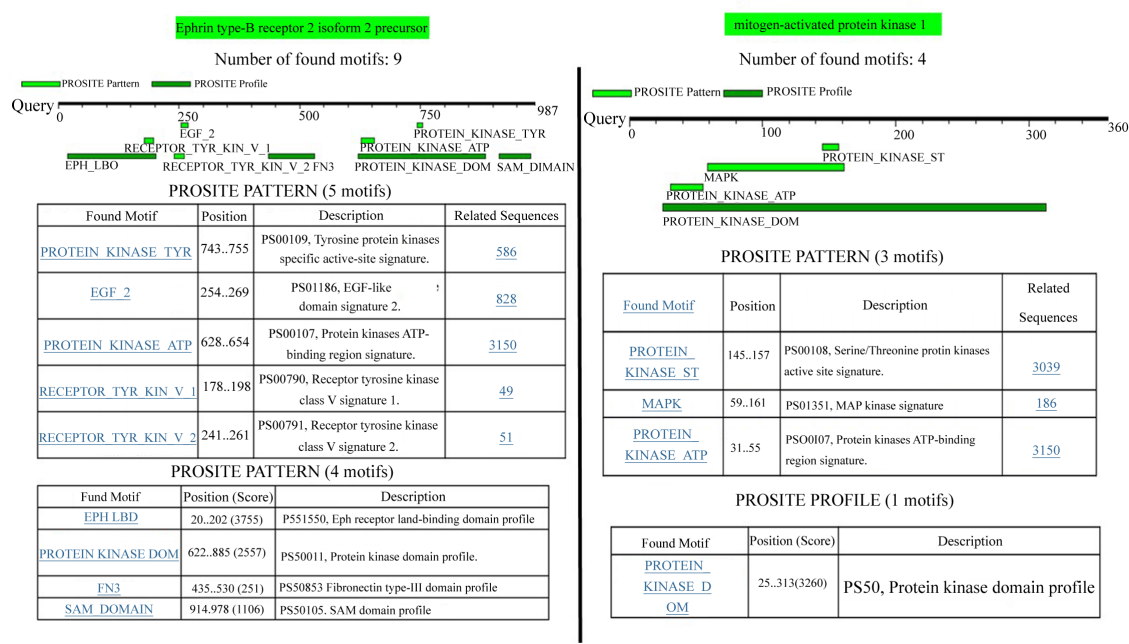

Figure 2. Left panel-No of motifs of ephrin type B receptor 2 isoform 2 precursor; Right panel-No of motifs of mitogen-activated protein kinase 1 . 


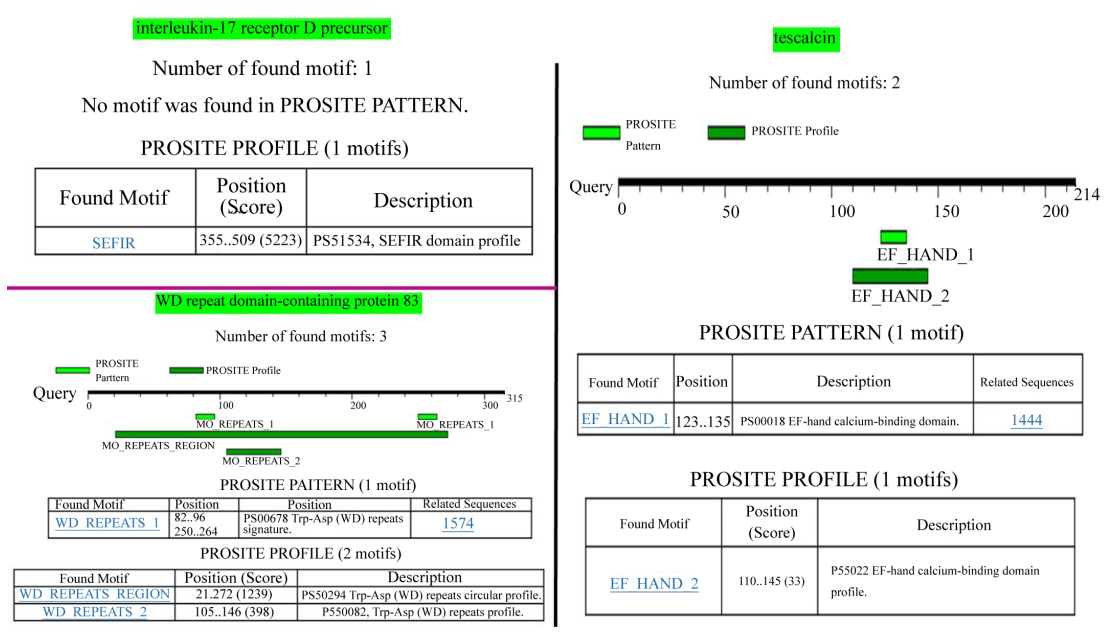

Figure 3. Left upper panel-No of motifs of interleukin-17 receptor D precursor; Left lower panel-No of motifs of WD repeat domain-containing protein 83 Right panel-No of motifs of tescalcin.
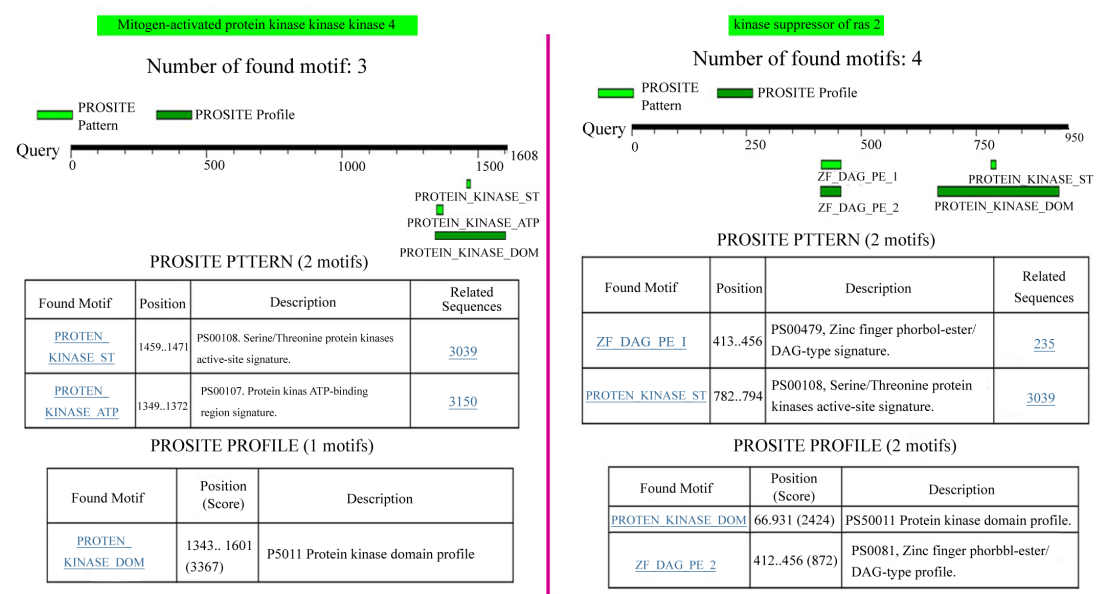

Figure 4. Left panel-No of motifs of mitogen activated protein kinase kinase kinase 4; Right panel: no of motifs of kinase suppressor of ras 2 .

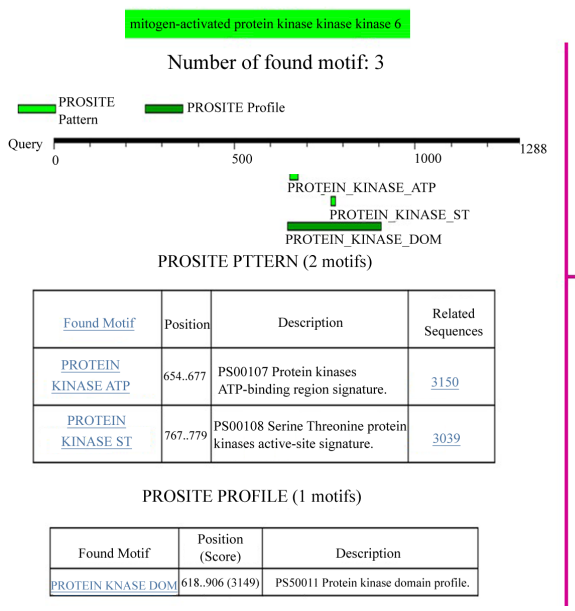

\section{UL16 binding prote 2}

Number of found motif: 0

No motif was found in PROSITE PATTERN.

No motif was found in PROSITE PROFILE.

Figure 5. Left panel-No of motifs of mitogen-activated protein kinase kinase 6; Right upper panel-No of motifs of UL16 binding protein 2; Right lower panel-No of motifs of UL16 binding protein 1 . 

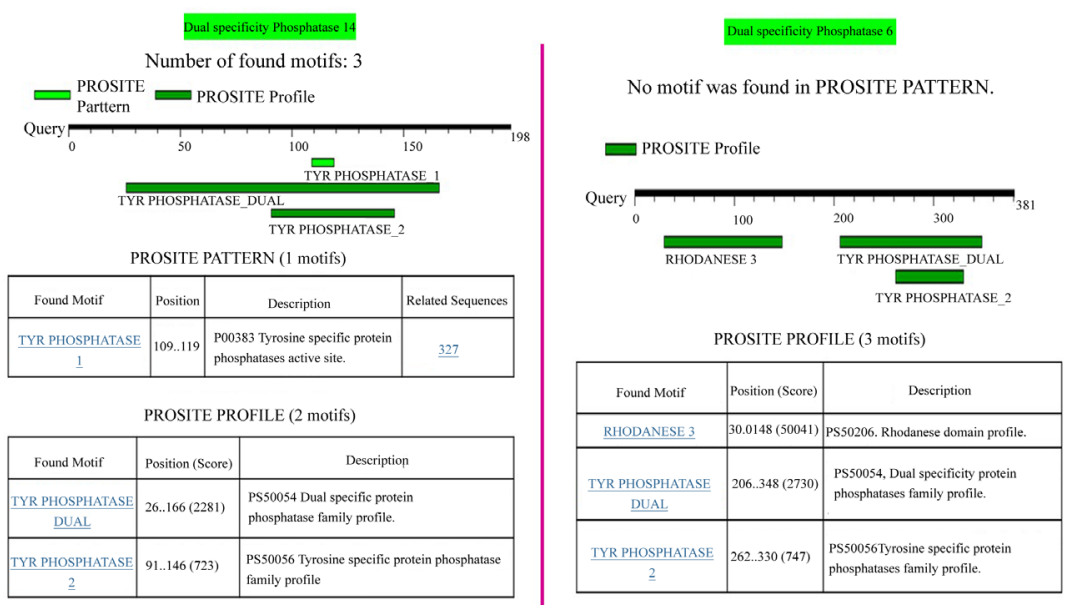

Figure 6. Left panel-No of motifs of dual specificity phosphatase 14; Right panel-No of motifs of dual specificity phosphatase.
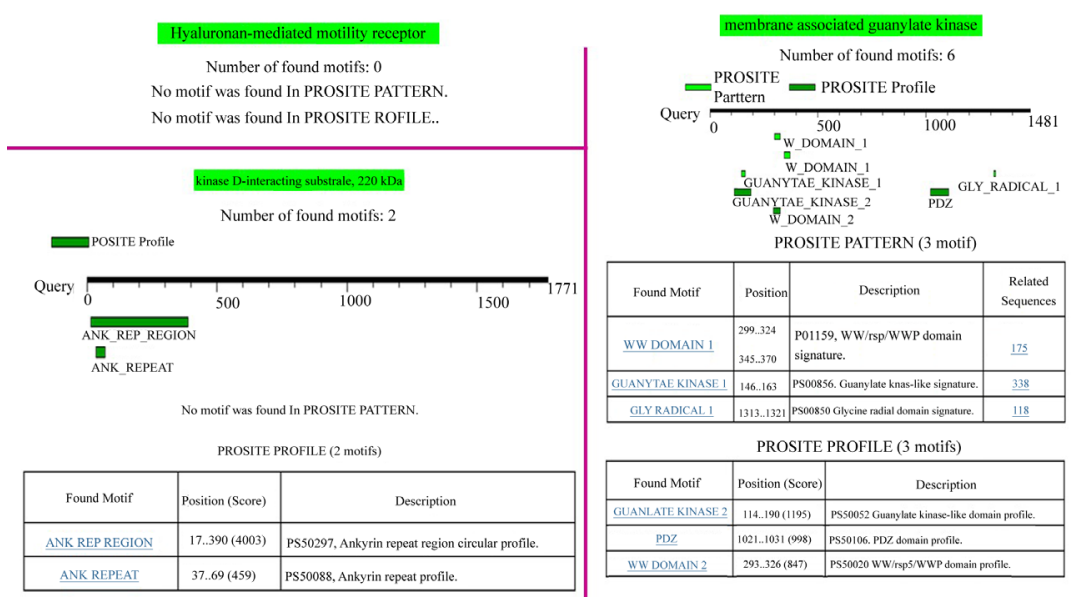

Figure 7. Left upper panel-No of motifs of hyaluronan-mediated motility receptor; Left lower panel-No of motifs of kinase D-interacting substrate of $220 \mathrm{kDa}$ Right panel-No of motifs of membrane associated guanylate kinase.

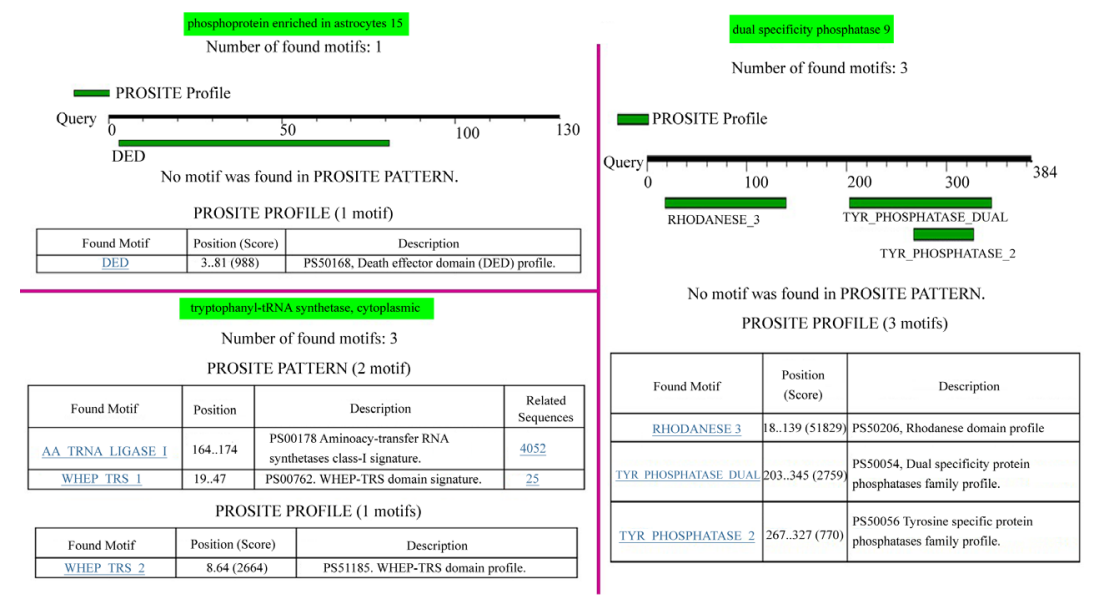

Figure 8. Left upper panel-No of motifs of phosphoprotein enriched in astrocytes 15; Left lower panel -No of motifs of tryptophanyl-tRNA synthetase, cytoplasmic Right panel-No of motifs of dual specificity phosphatase 9 . 


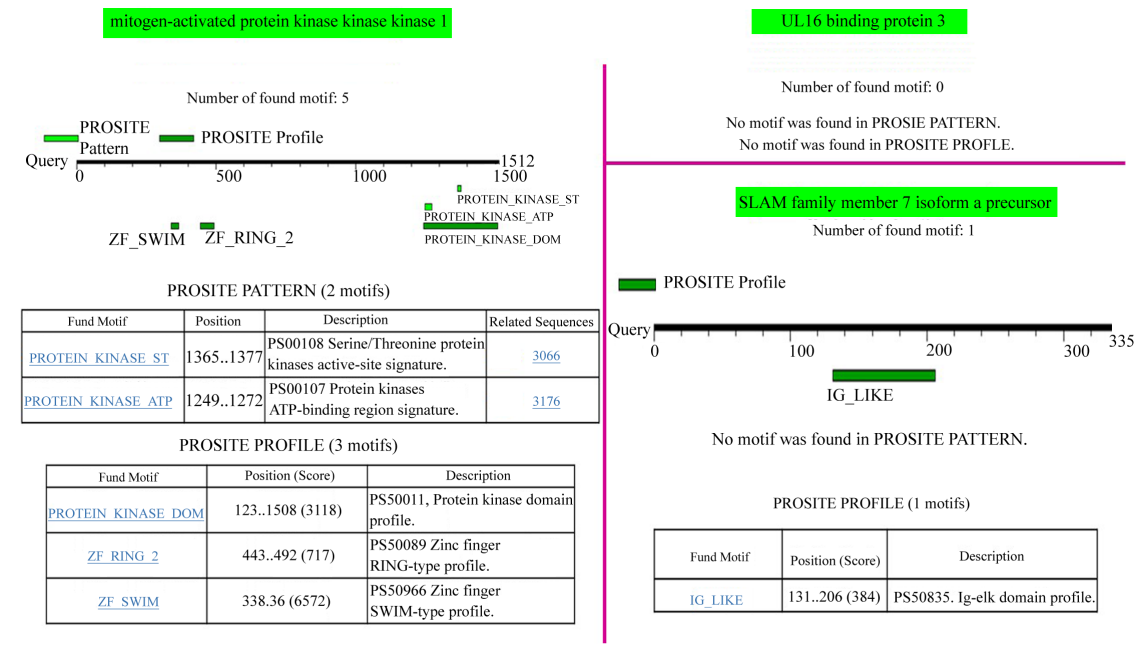

Figure 9. Left panel: No of motifs of mitogen-activated protein kinase kinase kinase 1; Right upper panel-No of motifs of UL16 binding protein 3; Right lower panel-No of motifs of SLAM family 7 isoform a precursor.

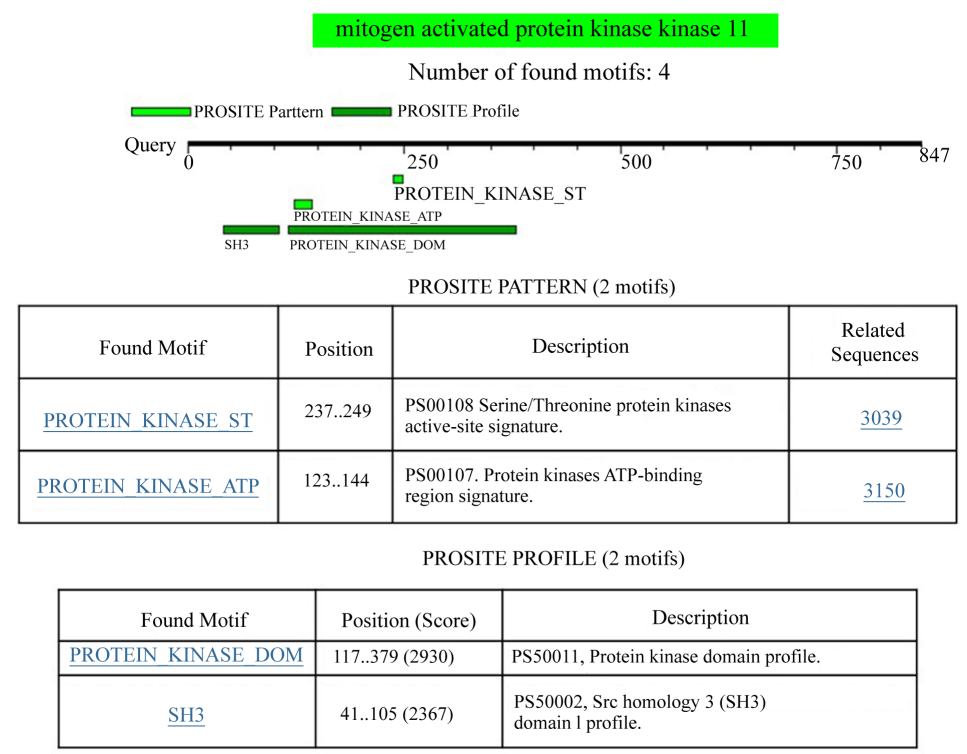

Figure 10. No of motifs of mitogen activated protein kinase kinase 11.

The secondary structure of 22 ERK interacting are mentioned in Table 2 and Figures 11-32 (Supplementary materials). Out of 22 ERK interacting proteins 12 proteins namely; EPHB2, IL17RD, KSR2, ULBP1, DUSP6, KIDINS220, MAGI3, DUSP9, MAP3K1, SLAMF7, MAP3K11 showed higher \% of random coil. Interestingly, when the dimensions of a number of proteins are compared, variations in the population of residual structure in the denatured state leads to deviations from ideal random coil behavior [9]. Moreover, these types of proteins do not attain conformational changes like secondary structure, tertiary structure and any other conformations [3]. Usually, in this situation, protein structure has been detectable in multidimensional magnetic resonance which depends on particular peptide-peptide communication. Likewise, parts of random coil appear merely 
Table 2. Secondary structure data of ERK interacting proteins.

\begin{tabular}{|c|c|}
\hline \multirow{10}{*}{ 1. Ephrin type-B receptor 2 isoform 2 precursor } & Alpha helix (Hh): 250 is $25.33 \%$ \\
\hline & 310 helix $(\mathrm{Gg}): 0$ is $0.00 \%$ \\
\hline & Pi helix (Ii): 0 is $0.00 \%$ \\
\hline & Beta bridge $(\mathrm{Bb}): 0$ is $0.00 \%$ \\
\hline & Extended strand (Ee): 211 is $21.38 \%$ \\
\hline & Beta turn (Tt): 54 is $5.47 \%$ \\
\hline & Bend region (Ss): 0 is $0.00 \%$ \\
\hline & Random coil (Cc): 472 is $47.82 \%$ \\
\hline & Ambiguous states (?): 0 is $0.00 \%$ \\
\hline & Other states: 0 is $0.00 \%$ \\
\hline \multirow{10}{*}{ 2. Mitogen-activated protein kinase 1} & Alpha helix (Hh): 178 is $49.44 \%$ \\
\hline & 310 helix $(\mathrm{Gg}): 0$ is $0.00 \%$ \\
\hline & Pi helix (Ii): 0 is $0.00 \%$ \\
\hline & Beta bridge $(\mathrm{Bb}): 0$ is $0.00 \%$ \\
\hline & Extended strand (Ee): 47 is $13.06 \%$ \\
\hline & Beta turn (Tt): 24 is $6.67 \%$ \\
\hline & Bend region (Ss): 0 is $0.00 \%$ \\
\hline & Random coil (Cc): 111 is $30.83 \%$ \\
\hline & Ambiguous states (?): 0 is $0.00 \%$ \\
\hline & Other states: 0 is $0.00 \%$ \\
\hline \multirow{10}{*}{ 3. Interleukin-17 receptor $D$ precursor } & Alpha helix (Hh): 223 is $30.18 \%$ \\
\hline & 310 helix $(\mathrm{Gg}): 0$ is $0.00 \%$ \\
\hline & Pi helix (Ii): 0 is $0.00 \%$ \\
\hline & Beta bridge $(\mathrm{Bb}): 0$ is $0.00 \%$ \\
\hline & Extended strand (Ee): 136 is $18.40 \%$ \\
\hline & Beta turn $(\mathrm{Tt}): 40$ is $5.41 \%$ \\
\hline & Bend region (Ss): 0 is $0.00 \%$ \\
\hline & Random coil (Cc): 340 is $46.01 \%$ \\
\hline & Ambiguous states (?): 0 is $0.00 \%$ \\
\hline & Other states: 0 is $0.00 \%$ \\
\hline \multirow{10}{*}{ 4. WD repeat domain-containing protein 83} & Alpha helix (Hh): 34 is $10.79 \%$ \\
\hline & 310 helix $(\mathrm{Gg}): 0$ is $0.00 \%$ \\
\hline & Pi helix (Ii): 0 is $0.00 \%$ \\
\hline & Beta bridge $(\mathrm{Bb}): 0$ is $0.00 \%$ \\
\hline & Extended strand (Ee): 125 is $39.68 \%$ \\
\hline & Beta turn (Tt): 43 is $13.65 \%$ \\
\hline & Bend region (Ss): 0 is $0.00 \%$ \\
\hline & Random coil (Cc): 113 is $35.87 \%$ \\
\hline & Ambiguous states (?): 0 is $0.00 \%$ \\
\hline & Other states: 0 is $0.00 \%$ \\
\hline \multirow{10}{*}{ 5. Tescalcin } & Alpha helix (Hh): 133 is $62.15 \%$ \\
\hline & 310 helix $(\mathrm{Gg}): 0$ is $0.00 \%$ \\
\hline & Pi helix (Ii): 0 is $0.00 \%$ \\
\hline & Beta bridge $(\mathrm{Bb}): 0$ is $0.00 \%$ \\
\hline & Extended strand (Ee): 11 is $5.14 \%$ \\
\hline & Beta turn (Tt): 7 is $3.27 \%$ \\
\hline & Bend region (Ss): 0 is $0.00 \%$ \\
\hline & Random coil (Cc): 63 is $29.44 \%$ \\
\hline & Ambiguous states (?): 0 is $0.00 \%$ \\
\hline & Other states: 0 is $0.00 \%$ \\
\hline
\end{tabular}




\section{Continued}

6. Mitogen-activated protein kinase kinasekinase 4

7. Kinase suppressor of Ras 2

8. Mitogen-activated protein kinase kinasekinase 6

9. UL16 binding protein 2

10. UL16 binding protein 1
Alpha helix (Hh): 756 is $47.01 \%$

310 helix (Gg): 0 is $0.00 \%$

Pi helix (Ii): 0 is $0.00 \%$

Beta bridge $(\mathrm{Bb}): 0$ is $0.00 \%$

Extended strand (Ee): 143 is $8.89 \%$

Beta turn (Tt): 65 is $4.04 \%$

Bend region (Ss): 0 is $0.00 \%$

Random coil (Cc): 644 is $40.05 \%$

Ambiguous states (?): 0 is $0.00 \%$

Other states: 0 is $0.00 \%$

Alpha helix (Hh): 254 is $26.74 \%$

310 helix (Gg): 0 is $0.00 \%$

Pi helix (Ii): 0 is $0.00 \%$

Beta bridge ( $\mathrm{Bb}): 0$ is $0.00 \%$

Extended strand (Ee): 128 is $13.47 \%$

Beta turn (Tt): 54 is $5.68 \%$

Bend region (Ss): 0 is $0.00 \%$

Random coil (Cc): 514 is $54.11 \%$

Ambiguous states (?): 0 is $0.00 \%$

Other states: 0 is $0.00 \%$

Alpha helix (Hh): 534 is $41.46 \%$

310 helix (Gg): 0 is $0.00 \%$

Pi helix (Ii): 0 is $0.00 \%$

Beta bridge ( $\mathrm{Bb})$ : 0 is $0.00 \%$

Extended strand (Ee): 174 is $13.51 \%$

Beta turn (Tt): 63 is $4.89 \%$

Bend region (Ss): 0 is $0.00 \%$

Random coil (Cc): 517 is $40.14 \%$

Ambiguous states (?): 0 is $0.00 \%$

Other states: 0 is $0.00 \%$

Alpha helix (Hh): 101 is $41.06 \%$

310 helix (Gg): 0 is $0.00 \%$

Pi helix (Ii): 0 is $0.00 \%$

Beta bridge $(\mathrm{Bb}): 0$ is $0.00 \%$

Extended strand (Ee): 38 is $15.45 \%$

Beta turn (Tt): 16 is $6.50 \%$

Bend region (Ss): 0 is $0.00 \%$

Random coil (Cc): 91 is $36.99 \%$

Ambiguous states (?): 0 is $0.00 \%$

Other states: 0 is $0.00 \%$

Alpha helix (Hh): 84 is $34.43 \%$

310 helix (Gg): 0 is $0.00 \%$

Pi helix (Ii): 0 is $0.00 \%$

Beta bridge ( $\mathrm{Bb}$ ): 0 is $0.00 \%$

Extended strand (Ee): 46 is $18.85 \%$

Beta turn (Tt): 18 is $7.38 \%$

Bend region (Ss): 0 is $0.00 \%$

Random coil (Cc): 96 is $39.34 \%$

Ambiguous states (?): 0 is $0.00 \%$

Other states: 0 is $0.00 \%$ 


\section{Continued}

11. Dual specificity phosphatase 14

12. Dual specificity phosphatase 6

Alpha helix (Hh): 73 is $36.87 \%$

310 helix (Gg): 0 is $0.00 \%$

Pi helix (Ii): 0 is $0.00 \%$

Beta bridge $(\mathrm{Bb}): 0$ is $0.00 \%$

Extended strand (Ee): 25 is $12.63 \%$

Beta turn (Tt): 10 is $5.05 \%$

Bend region (Ss): 0 is $0.00 \%$

Random coil (Cc): 90 is $45.45 \%$

Ambiguous states (?): 0 is $0.00 \%$

Other states: 0 is $0.00 \%$

Alpha helix (Hh): 128 is $33.60 \%$

310 helix (Gg): 0 is $0.00 \%$

Pi helix (Ii): 0 is $0.00 \%$

Beta bridge ( $\mathrm{Bb}): 0$ is $0.00 \%$

Extended strand (Ee): 59 is $15.49 \%$

Beta turn (Tt): 17 is $4.46 \%$

Bend region (Ss): 0 is $0.00 \%$

Random coil (Cc): 177 is $46.46 \%$

Ambiguous states (?): 0 is $0.00 \%$

Other states: 0 is $0.00 \%$

Alpha helix (Hh): 581 is $80.14 \%$

310 helix (Gg): 0 is $0.00 \%$

Pi helix (Ii): 0 is $0.00 \%$

Beta bridge $(\mathrm{Bb}): 0$ is $0.00 \%$

Extended strand (Ee): 18 is $2.48 \%$

Beta turn (Tt): 13 is $1.79 \%$

Bend region (Ss): 0 is $0.00 \%$

Random coil (Cc): 113 is $15.59 \%$

Ambiguous states (?): 0 is $0.00 \%$

Other states: 0 is $0.00 \%$

Alpha helix (Hh): 680 is $38.40 \%$

310 helix (Gg): 0 is $0.00 \%$

Pi helix (Ii): 0 is $0.00 \%$

Beta bridge $(\mathrm{Bb}): 0$ is $0.00 \%$

Extended strand (Ee): 200 is $11.29 \%$

14. Kinase $\mathrm{D}$-interacting substrate of $220 \mathrm{kDa}$

Beta turn (Tt): 98 is $5.53 \%$

Bend region (Ss): 0 is $0.00 \%$

Random coil (Cc): 793 is $44.78 \%$

Ambiguous states (?): 0 is $0.00 \%$

Other states: 0 is $0.00 \%$

Alpha helix (Hh): 223 is $15.06 \%$

310 helix (Gg): 0 is $0.00 \%$

Pi helix (Ii): 0 is $0.00 \%$

Beta bridge (Bb): 0 is $0.00 \%$

Extended strand (Ee): 229 is $15.46 \%$

Beta turn (Tt): 88 is $5.94 \%$

Bend region (Ss): 0 is $0.00 \%$

Random coil (Cc): 941 is $63.54 \%$

Ambiguous states (?): 0 is $0.00 \%$

Other states: 0 is $0.00 \%$ 


\section{Continued}

16. Phosphoprotein enriched in astrocytes 15

17. Tryptophanyl-tRNA synthetase, cytoplasmic

Alpha helix (Hh): 75 is $57.69 \%$

310 helix (Gg): 0 is $0.00 \%$

Pi helix (Ii): 0 is $0.00 \%$

Beta bridge $(\mathrm{Bb}): 0$ is $0.00 \%$

Extended strand (Ee): 8 is $6.15 \%$

Beta turn (Tt): 3 is $2.31 \%$

Bend region (Ss): 0 is $0.00 \%$

Random coil (Cc): 44 is $33.85 \%$

Ambiguous states (?): 0 is $0.00 \%$

Other states: 0 is $0.00 \%$

Alpha helix (Hh): 207 is $43.95 \%$

310 helix (Gg): 0 is $0.00 \%$

Pi helix (Ii): 0 is $0.00 \%$

Beta bridge ( $\mathrm{Bb})$ : 0 is $0.00 \%$

Extended strand (Ee): 48 is $10.19 \%$

Beta turn (Tt): 28 is $5.94 \%$

Bend region (Ss): 0 is $0.00 \%$

Random coil (Cc): 188 is $39.92 \%$

Ambiguous states (?): 0 is $0.00 \%$

Other states: 0 is $0.00 \%$

Alpha helix (Hh): 132 is $34.38 \%$

310 helix (Gg): 0 is $0.00 \%$

Pi helix (Ii): 0 is $0.00 \%$

Beta bridge ( $\mathrm{Bb}): 0$ is $0.00 \%$

Extended strand (Ee): 55 is $14.32 \%$

Beta turn (Tt): 26 is $6.77 \%$

Bend region (Ss): 0 is $0.00 \%$

Random coil (Cc): 171 is $44.53 \%$

Ambiguous states (?): 0 is $0.00 \%$

Other states: 0 is $0.00 \%$

Alpha helix (Hh): 538 is $35.58 \%$

310 helix (Gg): 0 is $0.00 \%$

Pi helix (Ii): 0 is $0.00 \%$

Beta bridge $(\mathrm{Bb}): 0$ is $0.00 \%$

Extended strand (Ee): 156 is $10.32 \%$

19. Mitogen-activated protein kinase kinasekinase 1

Beta turn (Tt): 66 is $4.37 \%$

Bend region (Ss): 0 is $0.00 \%$

Random coil (Cc): 752 is $49.74 \%$

Ambiguous states (?): 0 is $0.00 \%$

Other states: 0 is $0.00 \%$

Alpha helix (Hh): 88 is $36.07 \%$ 310 helix (Gg): 0 is $0.00 \%$

Pi helix (Ii): 0 is $0.00 \%$

Beta bridge ( $\mathrm{Bb}): 0$ is $0.00 \%$

Extended strand (Ee): 51 is $20.90 \%$

20. UL16 binding protein 3

Bend region (Ss): 0 is $0.00 \%$

Random coil (Cc): 87 is $35.66 \%$

Ambiguous states (?): 0 is $0.00 \%$

Other states: 0 is $0.00 \%$ 


\section{Continued}

\begin{tabular}{|c|c|}
\hline \multirow{10}{*}{ 21. SLAM family member 7 isoform a precursor } & Alpha helix (Hh): 68 is $20.30 \%$ \\
\hline & 310 helix $(\mathrm{Gg}): 0$ is $0.00 \%$ \\
\hline & Pi helix (Ii): 0 is $0.00 \%$ \\
\hline & Beta bridge $(\mathrm{Bb}): 0$ is $0.00 \%$ \\
\hline & Extended strand (Ee): 87 is $25.97 \%$ \\
\hline & Beta turn $(\mathrm{Tt}): 13$ is $3.88 \%$ \\
\hline & Bend region (Ss): 0 is $0.00 \%$ \\
\hline & Random coil (Cc): 167 is $49.85 \%$ \\
\hline & Ambiguous states (?): 0 is $0.00 \%$ \\
\hline & Other states: 0 is $0.00 \%$ \\
\hline \multirow{10}{*}{ 22. Mitogen-activated protein kinase kinasekinase 11} & Alpha helix (Hh): 229 is $27.04 \%$ \\
\hline & 310 helix $(\mathrm{Gg}): 0$ is $0.00 \%$ \\
\hline & Pi helix (Ii): 0 is $0.00 \%$ \\
\hline & Beta bridge $(\mathrm{Bb}): 0$ is $0.00 \%$ \\
\hline & Extended strand (Ee): 106 is $12.51 \%$ \\
\hline & Beta turn (Tt): 39 is $4.60 \%$ \\
\hline & Bend region (Ss): 0 is $0.00 \%$ \\
\hline & Random coil (Cc): 473 is $55.84 \%$ \\
\hline & Ambiguous states (?): 0 is $0.00 \%$ \\
\hline & Other states: 0 is $0.00 \%$ \\
\hline
\end{tabular}

Ephrin type-B receptor 2 isoform 2 precursor

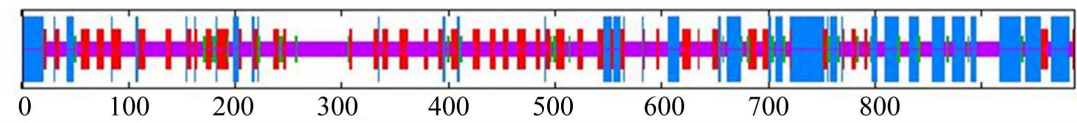

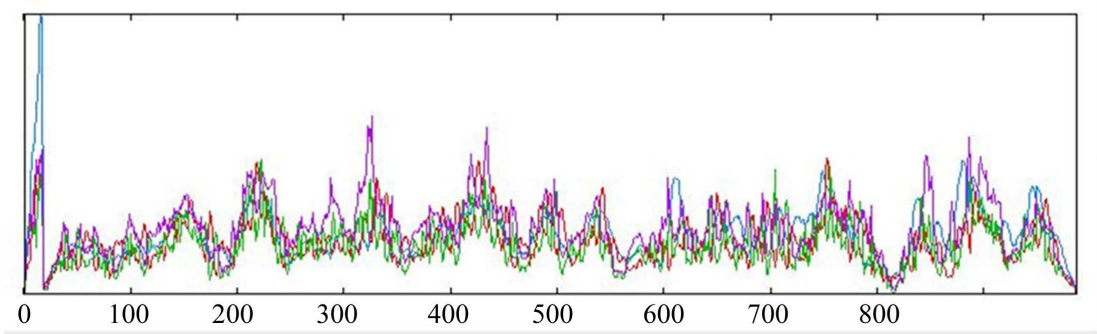

Figure 11. Secondary structure of EPHB2.

Mitogen-activated protein kinase 1
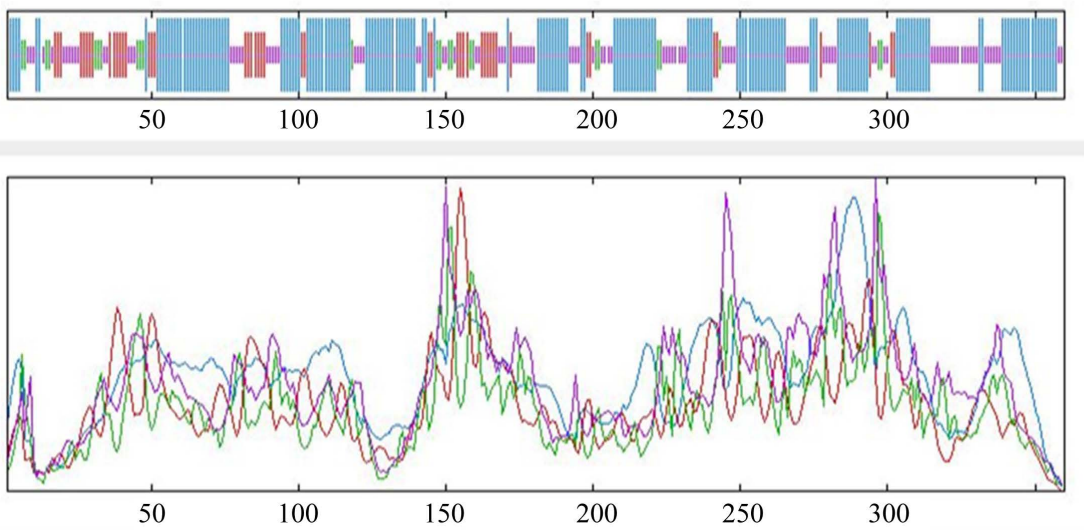

Figure 12. Secondary structure of MAPK1. 
Interlenkin-17 receptor D precursor
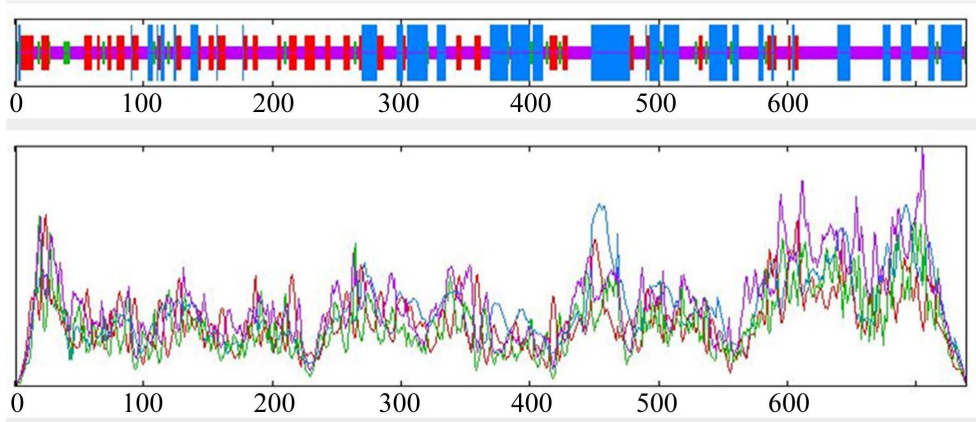

Figure 13. Secondary structure of IL17RD.

WD repeat domain-containing protein 83
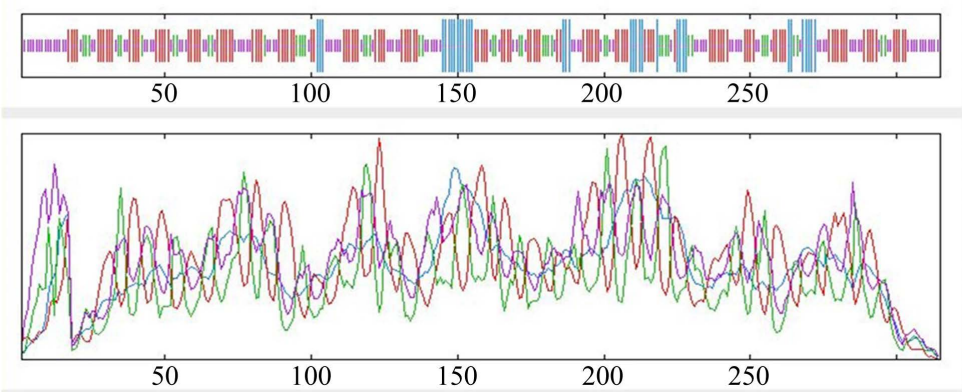

Figure 14. Secondary structure of WDR83.

Tescalcin
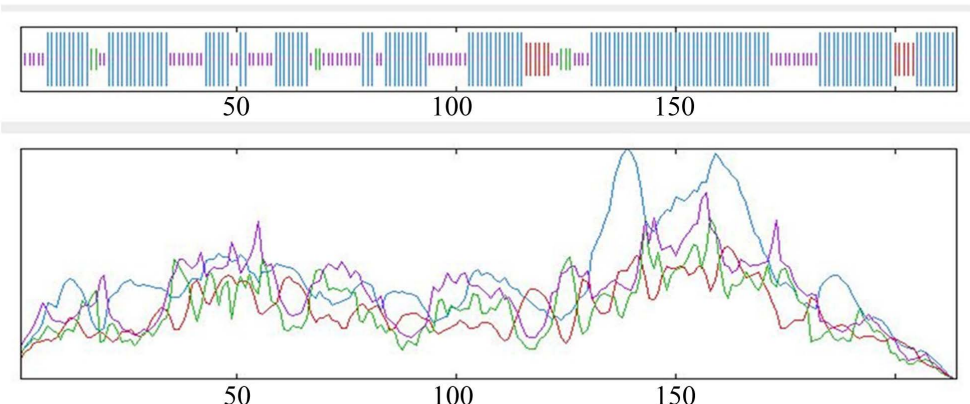

Figure 15. Secondary structure of TESC.

Mitogen-activated protein kinase kinase kinase 4
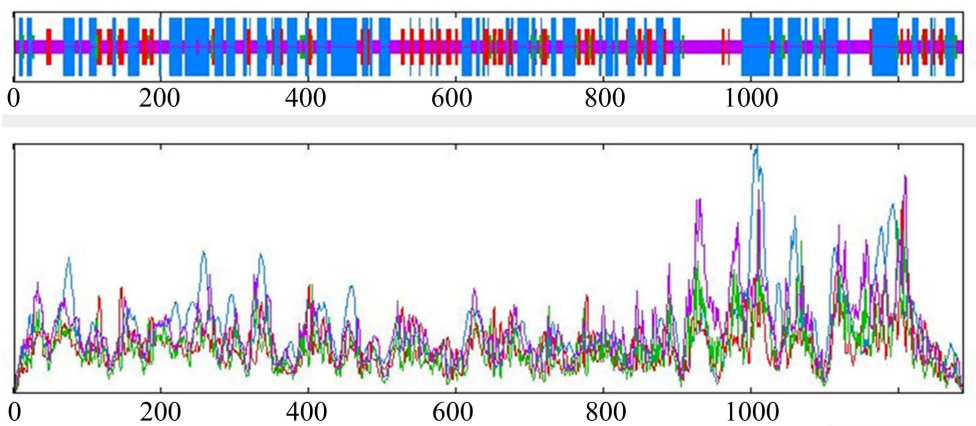

Figure 16. Secondary structure of MAP3K4. 
RecName: Fulll=Kinase suppressor of Ras 2; Short $=$ hKSR2
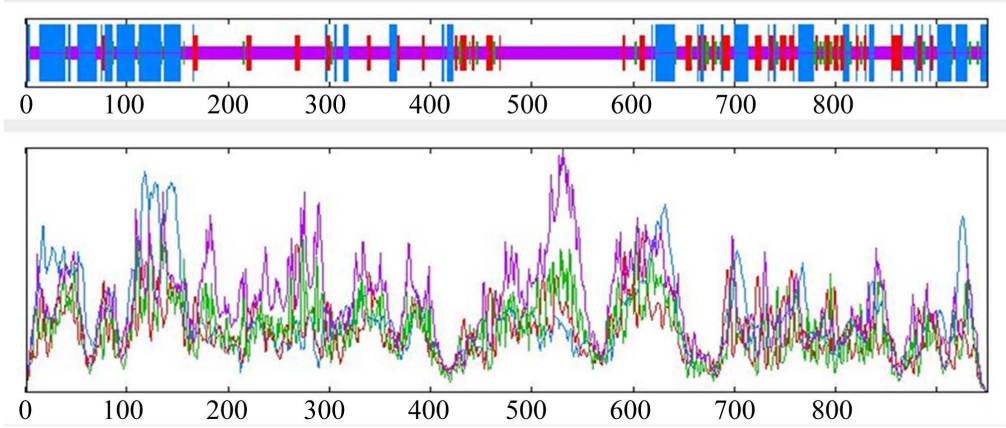

Figure 17. Secondary structure of KSR2.

Mitogen-activated protein kinase kinase kinase 6
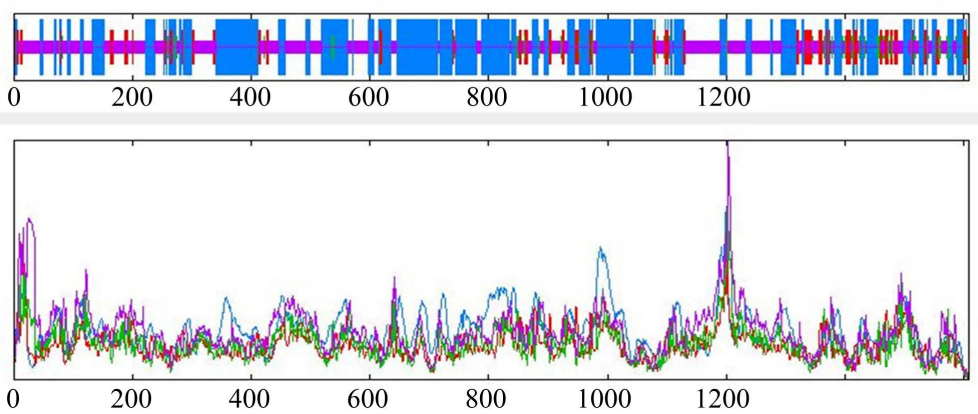

Figure 18. Secondary structure of MAP3K6.

UL16 binding protein 2
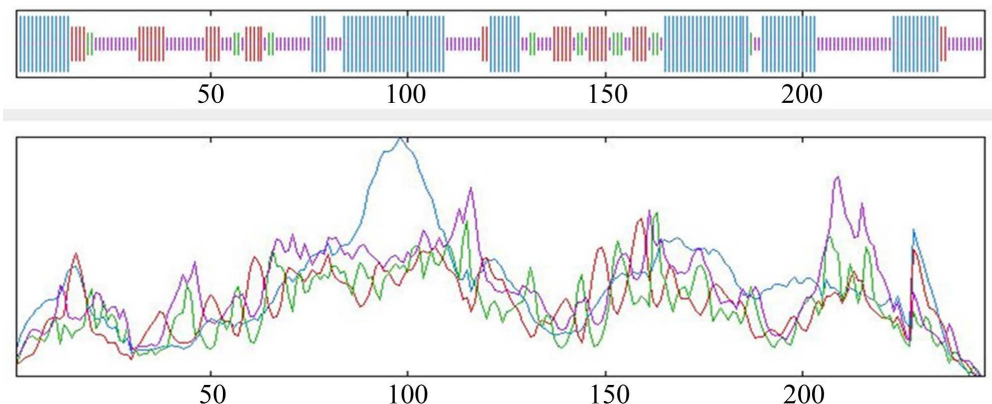

Figure 19. Secondary structure of ULBP2.

UL16 binding protein 1
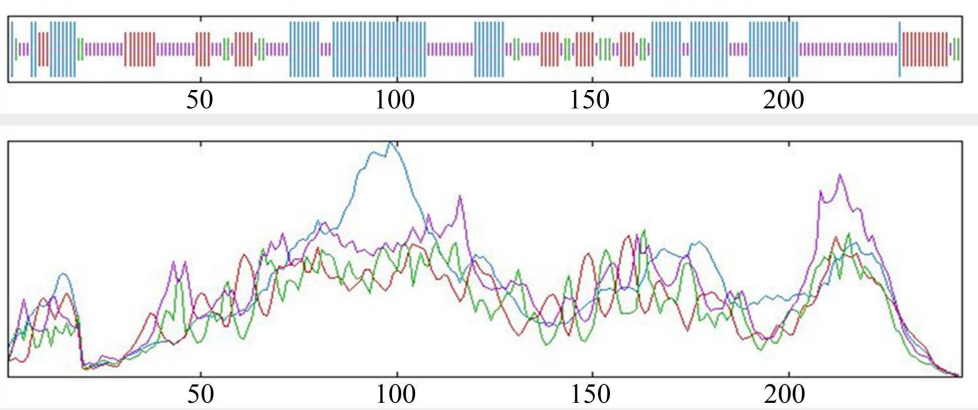

Figure 20. Secondary structure of ULBP1. 
Dual specificity phosphatase 14

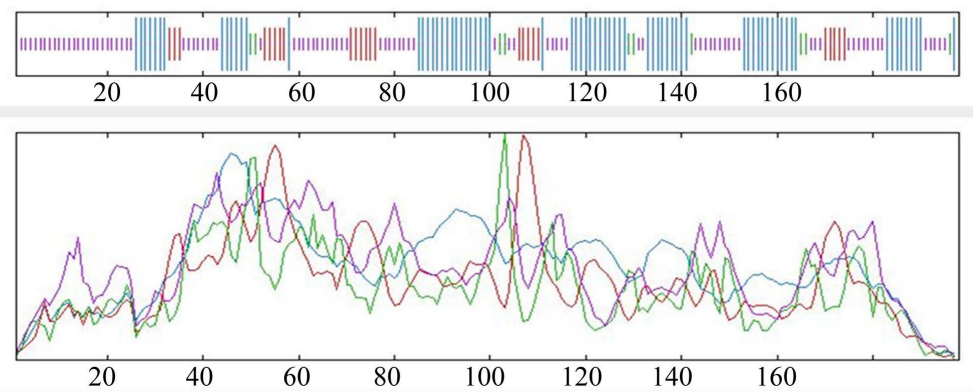

Figure 21. Secondary structure of DUSP14.

Dual specificity phosphatase 6
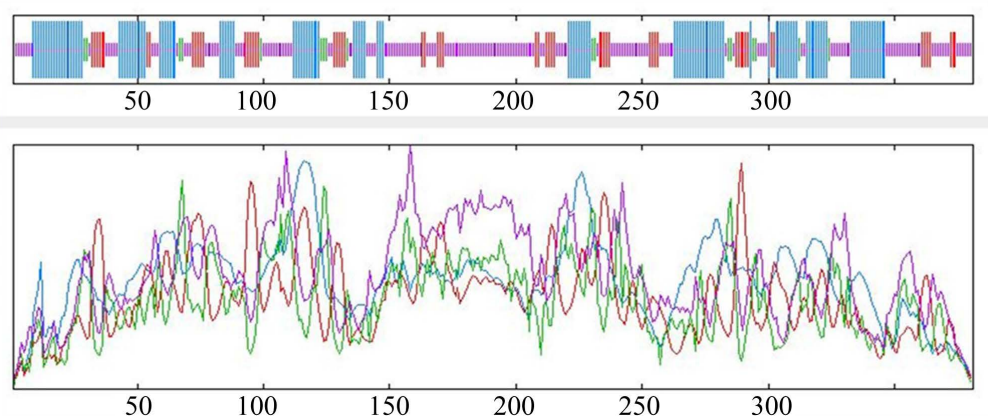

Figure 22. Secondary structure of DUSP6.

Hyaluronan-mediated motility receptor
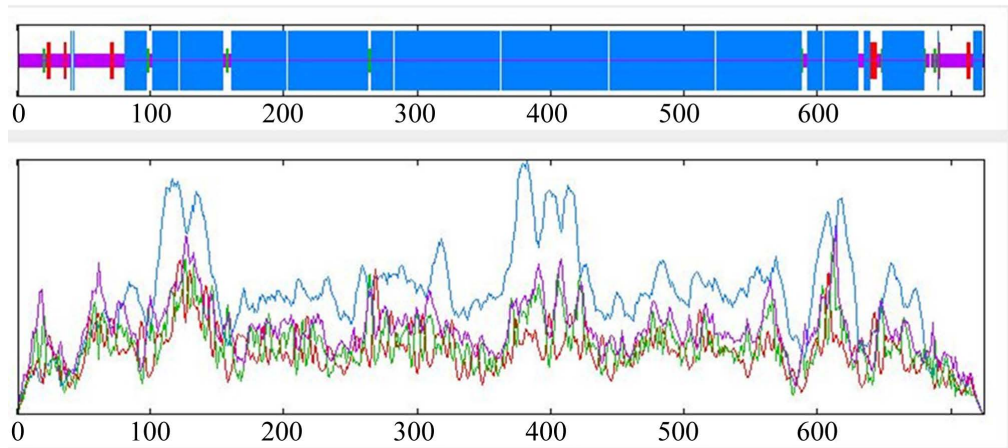

Figure 23. Secondary structure of RHAMM.

Kinase D-interacting substrate of $220 \mathrm{kDa}$
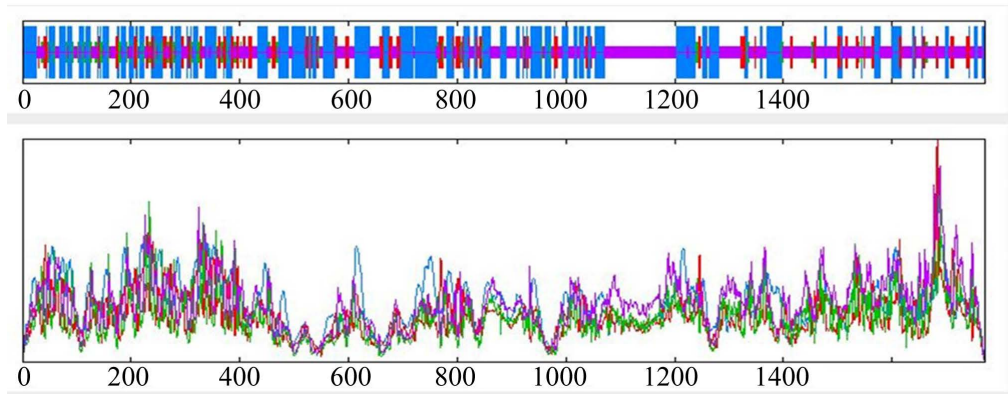

Figure 24. Secondary structure of KININS220. 
Membrane-associated guanylate kinase
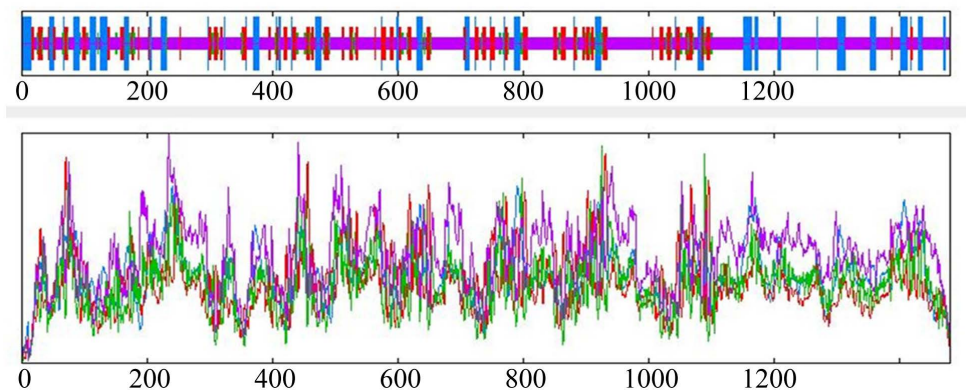

Figure 25. Secondary structure of MAGI3.

Phosphoprotein enriched in astrocytes 15
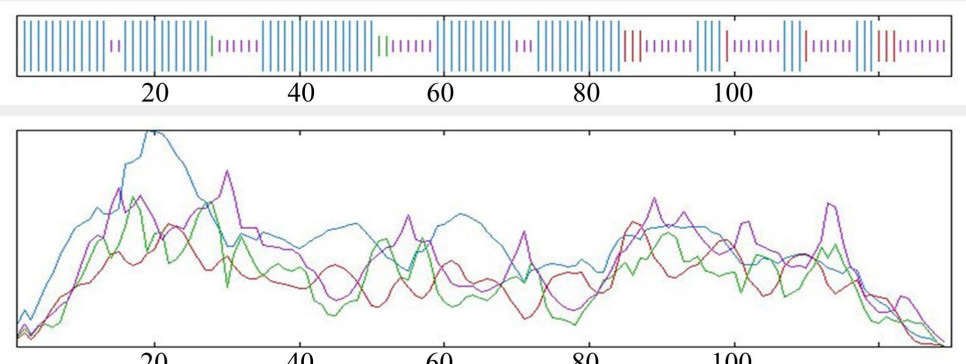

Figure 26. Secondary structure of PEA15.

Tryptophanyl-tRNA synthetase, cytoplasmic
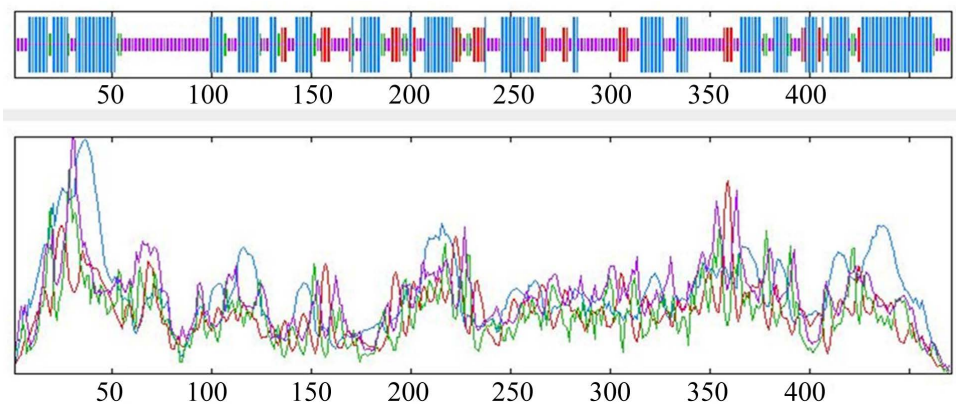

Figure 27. Secondary structure of WARS.

Dual specificity phosphatase 9
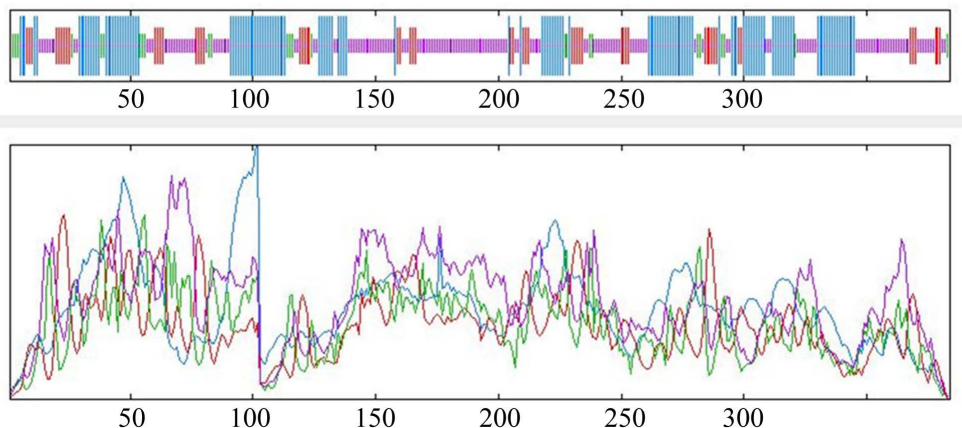

Figure 28. Secondary structure of DUSP9. 
Mitogen-activated protein kinase kinase kinase 1
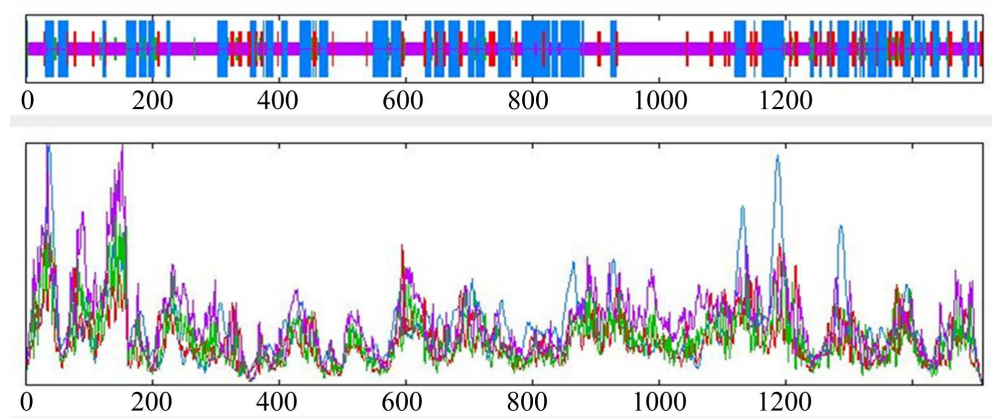

Figure 29. Secondary structure of MAP3K1.

UL1 6 binding protein 3

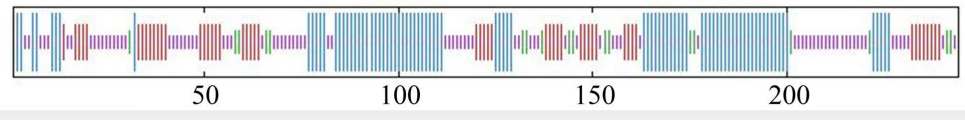

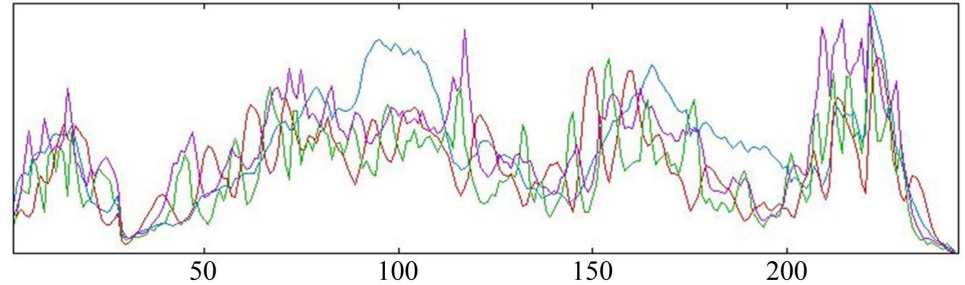

Figure 30. Secondary structure of ULBP3.

SLAM family member 7 isoform a precursor
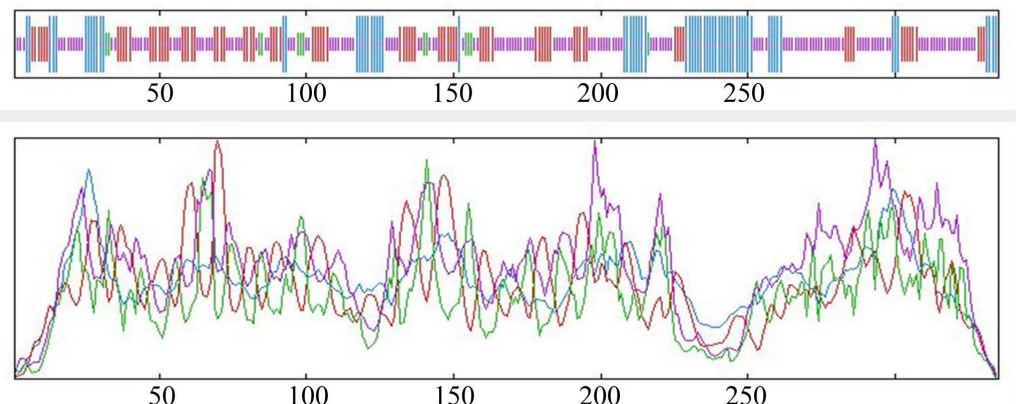

Figure 31. Secondary structure of SLAMF7.

Mitogen-activated protein kinase kinase kinase 11
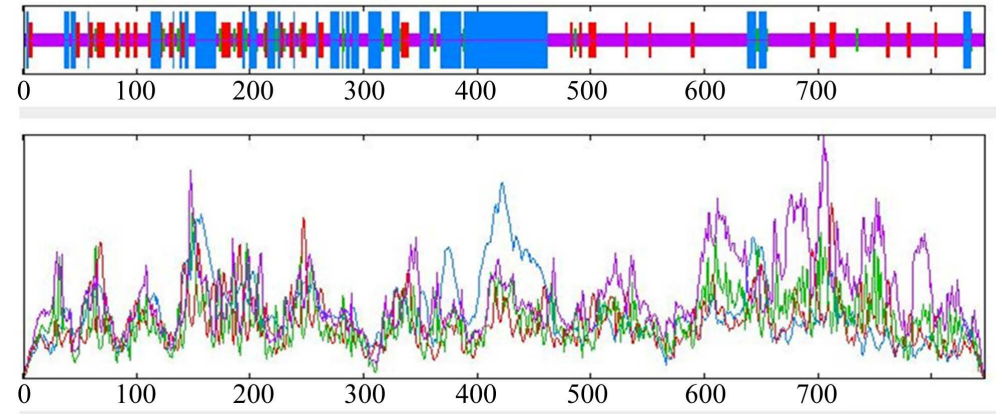

Figure 32. Secondary structure of MAP3K11. 
as a lack of "electron density" or contrast in the pictures generated in crystallography experiments. Circular dichroism is used to distinguish random coils. Moreover, the complete denaturation of protein lowers the percentage of random coil. We found that ERK interacting proteins were rich in random coil and alpha helix. Hence, ERK interacting proteins are not denatured and may perform various functions [3]. Moreover, 9 proteins namely MAPK1, TESC, MAP3K4, MAP3K6, ULBP2, RHAMM, WARS, ULBP3, PEA15, showed high percentage of alpha helix and WDR83 showed extended strand.

Long-term synaptic plasticity is the consequence of multiple gene expressions. ERK is one of those genes engaged in long-term memory and learning. A cascade of regulative genes has been recruited to particular genes including ERK in the process of long-term learning and memory formation [10] [11]. Moreover, chromatin remodeling is decisive for expression of genes [12]. ERK has been found to interact with a host of proteins to perform various cellular functions. Therefore, these proteins are important and involved in several diseases/disorders associated to the brain. Further, ERK interacting proteins have been reported to contain higher percentage of hydrophobic amino acid leucine [3].

Additionally, leucine works as an mTOR activator. mTOR also known as a mammalian target of rapamycin and FK 506-binding protein 12 rapamycin-associated protein 1 (FRAP1). FRAP1 is a kinase and encoded by the mTOR gene. mTOR links with other proteins and serves as a core component of two distinct protein complexes, mTOR complex 1 and mTOR complex 2. These both complexes work as a serine/threonine protein kinase. Being a core component of mTORC 2, mTOR works as a tyrosine protein kinase and promotes activation of insulin receptors as well as insulin like growth factor 1 receptors. In addition, mTOR modulates cell growth, proliferation and neuronal plasticity through protein synthesis regulation [13]. Specifically, mTOR controls the translation of a small segment of mRNAs containing a large secondary structure at their $5^{\prime}$ UTR or oligopyrimidine at their 5' ends (TOP mRNAs) [14] [15]. This kinase can be triggered by distinct extracellular signals and controls protein synthesis at initiation stage primarily by phosphorylation of at least two downstream targets mainly p70S6 kinase (p70S6 K) and eukaryotic initiation factor 4E-binding proteins [16].

In neurons, mTOR has been reported in the synaptic region and mediates synthesis of locally-translated proteins. Also, mTOR is up-regulated in an activity dependent manner and critical for different forms of synaptic plasticity including long-term potentiation (LTP) [17] [18]. Nonetheless, little is known about the extracellular signals activated by training and trigger mTOR for protein synthesis regulation during memory consolidation. However, mTOR is required for memory formation in many learning tasks [19] [20]. BDNF induces rapamycin-sensitive synaptic potentiation [15] [17] and regulates translation of 5' TOP mRNA encoded proteins at dendrites through an mTOR-dependent pathway [15] [19] [21] [22]. Taken together, it can be concluded that leucine rich food supplement can be helpful for learning and memory. 
In addition, ERK interacting proteins showed higher serine/threonine kinases active site signature and protein kinase ATP binding region signature domain commonly in ProPat. They showed protein kinase domain, dual specificity protein phosphatase and tyrosine specific protein phosphatase family domain in ProPro. Several studies have suggested that learning and memory are mediated by neuronal plasticity, strengthening of LTP [23], synaptogenesis [24], modulation of intrinsic excitability [25] and adult neurogenesis [26]. All the above-mentioned processes may require protein kinases (enzymes that transfer phosphate groups to side chains of particular amino acids (serine/threonine or tyrosine) of target proteins). Moreover, phosphorylation at one or more sites of a protein has been involved to alter the conformation of target proteins affecting their functions or interaction with other proteins [27]. Analysis of the Allen Mouse Brain Atlas suggests that at least 260 kinases are expressed in adult mouse brain. The involvement of these kinases has been implicated in learning and memory. These findings support our results as an ERK interacting proteins showed richness in protein kinase domain. Such studies are helpful to understand ERK mediated cell singling pathways including neurodegenerative diseases.

Table 3. ERK interacting proteins and their motifs.

\begin{tabular}{|c|c|c|c|c|}
\hline S.N. & $\begin{array}{l}\text { NCBI Reference } \\
\text { Sequence }\end{array}$ & $\begin{array}{l}\text { Gene } \\
\text { Name }\end{array}$ & Protein Name & No of found domain \\
\hline 1 & NP_004433 & ЕPHB2 & Ephrin type-B receptor 2 isoform 2 precursor & Total $=9($ ProPat -5, ProPro -4$) /$ motifs \\
\hline 2 & NP_620407.1 & MAPK1 & Mitogen-activated protein kinase 1 & Total $=4$ (ProPat -3, ProPro- 1$)$ \\
\hline 3 & NP_060033.3 & IL17RD & Interleukin-17 receptor D precursor & Total $=1($ ProPat-none, ProPro- 1$)$ \\
\hline 4 & NP_001093207.1 & WDR83 & WD repeat domain-containing protein 83 & Total $=3$ (ProPat -1, ProPro- 2$)$ \\
\hline 5 & AAH15221.1 & TESC & Tescalcin & Total $=2($ ProPat -1, ProPro -1$)$ \\
\hline 6 & AAI36277.1 & MAP3K4 & Mitogen-activated protein kinase kinasekinase 4 & Total $=3($ ProPat -2, ProPro- 1$)$ \\
\hline 7 & Q6VAB6.2 & KSR2 & Rec Name: Full = Kinase suppressor of Ras 2; Short $=$ hKSR2 & Total $=4$ (ProPat -2, ProPro-2) \\
\hline 8 & EAX07758.1 & MAP3K6 & Mitogen-activated protein kinase kinasekinase 6 & Total $=3($ ProPat -2, ProPro -1$)$ \\
\hline 9 & AAQ89028.1 & ULBP2 & UL16 binding protein 2 & Total = none $($ ProPat-nil, ProPro-none $)$ \\
\hline 10 & AAK13081.1 & ULBP1 & UL16 binding protein 1 & Total $=$ none $($ ProPat-none, ProPro-none $)$ \\
\hline 11 & CAG38739.1 & DUSP14 & Dual specificity phosphatase 14 & Total $=3$ (ProPat -1, ProPro -2$)$ \\
\hline 12 & BAA34369.1 & DUSP6 & dual specificity phosphatase 6 & Total $=3$ (ProPat-none, ProPro- 3 ) \\
\hline 13 & AAI08905.1 & RHAMM & Hyaluronan-mediated motility receptor & Total = none (ProPat-nil, ProPro-none $)$ \\
\hline 14 & NP_065789.1 & KIDINS220 & Kinase D-interacting substrate of $220 \mathrm{kDa}$ & Total $=2($ ProPat-none, ProPro-2) \\
\hline 15 & NP_001136254.1 & MAGI3 & Membrane-associated guanylate kinase & Total $=6$ (ProPat -3, ProPro -3$)$ \\
\hline 16 & CAG46533.1 & PEA15 & Phosphoprotein enriched in astrocytes 15 & Total $=1($ ProPat-none, ProPro- 1$)$ \\
\hline 17 & AAH95453.1 & WARS & Tryptophanyl-tRNA synthetase, cytoplasmic & Total $=4$ (ProPat -3, ProPro -1$)$ \\
\hline 18 & AAH60837.1 & DUSP9 & Dual specificity phosphatase 9 & Total $=3$ (ProPat-none, ProPro- 3 ) \\
\hline 19 & NP_005912.1 & MAP3K1 & Mitogen-activated protein kinase kinasekinase 1 & Total $=5($ ProPat -2, ProPro -3$)$ \\
\hline 20 & AAK13083.1 & ULBP3 & UL16 binding protein 3 & Total $=$ none $($ ProPat - nil, ProPro-none $)$ \\
\hline 21 & NP_067004.3 & SLAMF7 & SLAM family member 7 isoform a precursor & Total $=1$ (ProPat-none, ProPro- 1$)$ \\
\hline 22 & NP_002410.1 & MAP3K11 & Mitogen-activated protein kinase kinasekinase 11 & Total $=4$ (ProPat -2, ProPro -2$)$ \\
\hline
\end{tabular}




\section{Conclusion}

Majority of the ERK interacting proteins are rich in random coil and alpha helix \% (Table 2). Further, most of interacting proteins have serine/threonine kinases active site signature and protein kinase ATP binding region. In addition, few have commonly dual specificity protein phosphatase family and tyrosine specific protein phosphatase domains (Table 3). Such study may be helpful to design engineered molecules for regulating ERK dependent pathways in disease condition.

\section{Acknowledgements}

VP acknowledges Science and Engineering Research Board (SERB), Government of India for providing financial support (SB/YS/LS-200/2013). KK acknowledges SERB for Junior Research Fellowship and Indian Council of Medical Research (ICMR) for Senior Research Fellowship (Letter No. 45/72/1 8-PHA/BMS/OL).

\section{Conflicts of Interest}

Authors declare no conflict of interest in any issue.

\section{References}

[1] Biondi, M.R. and Nebreda, R.A. (2003) Signalling Specificity of Ser/Thr Protein Kinases through Docking-Site-Mediated Interactions. Biochemical Journal, 372, 1-13. https://doi.org/10.1042/bj20021641

[2] Fantz, D.A., Jacobs, D., Glossip, D. and Kornfeld, K. (2001) Docking Sites on Substrate Proteins Direct Extracellular Signal Regulated Kinase to Phosphorylate Specific Residues. Journal of Biological Chemistry, 276, 27256-27265. https://doi.org/10.1074/jbc.M102512200

[3] Kurrey, K. and Paramanik, V. (2018) Identification and Physiochemical Analysis of ERK Interacting Proteins Using Bio-Computational Tools. World Journal of Neuroscience, 8, 303-313. https://doi.org/10.4236/wjns.2018.82024

[4] Ebisuya, M., Kondoh, K. and Nishida, E. (2005) The Duration, Magnitude and Compartmentalization of ERK MAP Kinase Activity: Mechanisms for Providing Signaling Specificity. Journal of Cell Science, 118, 2997-3002. https://doi.org/10.1242/jcs.02505

[5] Taylor, S.S. and Radzio-Andzelm, E. (1994) Three Protein Kinase Structures Define a Common Motif. Structure, 2, 345-355. https://doi.org/10.1016/S0969-2126(00)00036-8

[6] Kleywegt, G.J. (1999) Recognition of Spatial Motifs in Protein Structures. Journal of Molecular Biology, 285, 1887-1897. https://doi.org/10.1006/jmbi.1998.2393

[7] Rajasekaran, S.,Balla, S., Gradie, P., Gryk, M.R., Kadaveru, K., Kundeti, V., et al. (2009) Minimotif Miner 2nd Release: A Database and Web System for Motif Search. Nucleic Acid Research, 37, D185-D190. https://doi.org/10.1093/nar/gkn865

[8] Sigrist, C.J., Cerutti, L., Hulo, N., Gattiker, A., Falquet, L., Pagni, M., et al. (2002) PROSITE: A Documented Database Using Patterns and Profiles as Motif Descriptors. Briefings in Bioinformatics, 3, 265-274. https://doi.org/10.1093/bib/3.3.265

[9] Kohn, J.E., Millett, I.S., Jacob, J., Zagrovic, B., Zagrovic, B., Dillon, T.M., Cingel, N., 
et al. (2004) Random-Coil Behavior and the Dimensions of Chemically Unfolded Proteins. Proceedings of the National Academy of Sciences of the United States of America, 101, 12491-112496. https://doi.org/10.1073/pnas.0403643101

[10] Goelet, P., Castellucci, F.V., Schacher, S. and Kandel, R.E. (1986) The Long and the Short of Long-Term Memory-A Molecular Framework. Nature, 322, 419-422.

https://doi.org/10.1038/322419a0

[11] Taubenfeld, M.S., Milekic, H.M., Monti, B. and Alberini, M.C. (2001) The Consolidation of New but Not Reactivated Memory Requires Hippocampal C/EBP $\beta$. Nature Neuroscience, 4, 813-818. https://doi.org/10.1038/90520

[12] McClung, C.A. and Nestler, E.J. (2008) Neuroplasticity Mediated by Altered Gene Expression. Neuropsychopharmacology, 33, 3-17. https://doi.org/10.1038/sj.npp.1301544

[13] Hay, N. and Sonenberg N. (2004) Upstream and Downstream of mTOR. Genes and Development, 18, 1926-1945. https://doi.org/10.1101/gad.1212704

[14] Ruvinsky, I. and Meyuhas, O. (2006) Ribosomal Protein S6 Phosphorylation: From Protein Synthesis to Cell Size. Trends in Biochemical Sciences, 31, 342-348. https://doi.org/10.1016/j.tibs.2006.04.003

[15] Slipczuk, L., Bekinschtein, P., Katche, C., Cammarota, M.I., Izquierdo, I. and Medina, J.H. (2009). BDNF Activates mTOR to Regulate GluR1 Expression Required for Memory Formation. PLoS ONE, 4, e6007. https://doi.org/10.1371/journal.pone.0006007

[16] Costa-Mattioli, M., Sossin, W.S., Klann, E. and Sonenberg, N. (2009) Translational Control of Long-Lasting Synaptic Plasticity and Memory. Neuron, 61, 10-26. https://doi.org/10.1016/j.neuron.2008.10.055

[17] Tang, S.J., Reis, G., Kang, H., Gingras, A., Sonenberg, N. and Schuman, E.M. (2002) A Rapamycin-Sensitive Signaling Pathway Contributes to Long-Term Synaptic Plasticity in the Hippocampus. Proceedings of the National Academy of Sciences of the United States of America, 99, 467-472. https://doi.org/10.1073/pnas.012605299

[18] Cammalleri, M., Lutjens, R., Berton, F., King, R.A., Simpson, C., Francesconi, W., et al. (2003) Time-Restricted Role for Dendritic Activation of the mTOR-p70 ${ }^{\mathrm{S} 6 \mathrm{~K}}$ Pathway in the Induction of Late-Phase Long-Term Potentiation in the CA1. Proceedings of the National Academy of Sciences of the United States of America, 100, 14368-14373. https://doi.org/10.1073/pnas.2336098100

[19] Bekinschtein, P., Katche, C., Slipczuk, L.N., Igaz, M.L., Cammarota, M., Izquierdo, I., et al. (2007) TOR Signaling in the Hippocampus Is Necessary for Memory Formation. Neurobiology of Learning and Memory, 87, 303-307. https://doi.org/10.1016/j.nlm.2006.08.007

[20] Lu, Y., Christian, K. and Lu, B. (2008) BDNF: A Key Regulator for Protein Synthesis-Dependent LTP and Long-Term Memory? Neurobiology of Learning and Memory, 89, 312-323. https://doi.org/10.1016/j.nlm.2007.08.018

[21] Dudai, Y. and Eisenberg, M. (2004) Rites of Passage of the Engram: Reconsolidation and the Lingering Consolidation Hypothesis. Neuron, 44, 93-100. https://doi.org/10.1016/j.neuron.2004.09.003

[22] Takei, N., Inamura, N., Kawamura, M., Namba, H., Hara, K., Yonezawa, K., et al. (2004) Brain-Derived Neurotrophic Factor Induces Mammalian Target of Rapamycin-Dependent Local Activation of Translation Machinery and Protein Synthesis in Neuronal Dendrites. Journal of Neuroscience, 24, 9760-9769. https://doi.org/10.1523/JNEUROSCI.1427-04.2004

[23] Giese, K.P. (2012) Long-Term Potentiation and Memory. In: Giese, K.P., Ed., 
Memory Mechanism and Health Disease: Mechanistic Basis of Memory, World Scientific, Singapore, 1-17. https://doi.org/10.1142/9789814366700 0001

[24] Stewart, M.G. and Popov, V.I. (2012) Structural Synaptic and Dendritic Spine Plasticity in the Hippocampus. In: Giese, K.P., Ed., Memory Mechanisms in Health and Disease, World Scientific Publishing, Singapore, 19-52.

https://doi.org/10.1142/9789814366700 0002

[25] Papoutsi, A., Sidiropoulou, K., Cutsuridis, V. and Poirazi, P. (2013) Induction and Modulation of Persistent Activity in a Layer V PFC Microcircuit Model. Frontiers of Neural Circuits, 7, 161. https://doi.org/10.3389/fncir.2013.00161

[26] Stone, S.S.D., Frankland, P.W. (2012) Adult Hippocampal Neurogenesis and Memory. In: Giese, K.P., Ed., Memory Mechanisms in Health and Disease, World Scientific Publishing, Singapore, 81-146. https://doi.org/10.1142/9789814366700 0004

[27] Kim, S.Y., Hwang, G.S., Han, H. and Cho, M. (2011) Phosphorylation Alters Backbone Conformational Preferences of Serine and Threonine Peptides. Proteins: Structure, Function, and Bioinformatics, 79, 3155-3165.

https://doi.org/10.1002/prot.23148 